\title{
Concerns of quality, utility, and reliability of laparoscopic gastrectomy for gastric cancer in public video sharing platform
}

\author{
Shun Zhang ${ }^{1,2}$, Tetsu Fukunaga ${ }^{1}$, Shinichi Oka ${ }^{1}$, Hajime Orita ${ }^{1}$, Sanae Kaji ${ }^{1}$, Yukinori Yube ${ }^{1}$, \\ Suguru Yamauchi ${ }^{1}$, Yoshinori Kohira ${ }^{1}$, Hiroyuki Egawa ${ }^{1}$ \\ ${ }^{1}$ Department of Gastrointestinal Surgery, Shanghai East Hospital (East Hospital Affiliated to Tongji University), Shanghai 200120, China; \\ ${ }^{2}$ Department of Gastroenterology and Minimally Invasive Surgery, Juntendo University Hospital, Tokyo, Japan \\ Contributions: (I) Conception and design: S Zhang, T Fukunaga; (II) Administrative support: H Orita, T Fukunaga; (III) Provision of study materials \\ or patients: S Zhang, H Orita, S Oka; (IV) Collection and assembly of data: S Zhang, S Oka, S Kaji; (V) Data analysis and interpretation: S Zhang, Y \\ Kohira, H Egawa; (VI) Manuscript writing: All authors; (VII) Final approval of manuscript: All authors. \\ Correspondence to: Tetsu Fukunaga, MD, PhD. Department of Gastroenterology and Minimally Invasive Surgery, Juntendo University Hospital, 3-1-3 \\ Hongo, Bunkyo-ku, Tokyo, Japan. Email: t2fukunaga@juntendo.ac.jp.
}

Background: The rapid expansion of laparoscopic gastrectomy (LG) for gastric cancer has generated interest among surgeons. The adequate dissemination of correct information about such advanced laparoscopic surgery can certainly be useful for surgeons and trainees. Online video resources such as YouTube are frequently used for education. This study aimed to evaluate the quality, utility, and completeness of LG videos for gastric cancer on the video website YouTube.

Methods: The terms "laparoscopic gastrectomy" and "gastric cancer" were searched on YouTube on August 16, 2019. The first 100 videos in three sorting categories (website's default setting, view count, and length of duration) were checked by two experienced surgeons. The popularity was evaluated with the video power index (VPI). The reliability was measured using the Fournal of American Medical Association (ZAMA) benchmark criteria. The educational value and completeness were evaluated with a checklist developed by the researchers.

Results: A total of 102 videos were analyzed. Laparoscopic distal gastrectomy (LDG) and laparoscopic total gastrectomy were the most frequently recorded techniques. Lymph node (LN) dissection was the most frequently covered topic (89.2\%), followed in descending order by GI reconstruction (87.3\%). The mean VPI, JAMA benchmark score and completeness score of all videos were 2.63, 1.94 and 8.53, respectively. The types of sources were as follows: private users, 73 (71.6\%); academic institutions, 20 (19.6\%); and others, 9 (8\%). A total of 97 videos with an identifiable primary surgeon originated from eighteen different countries.

Conclusions: Laparoscopic videos represented by YouTube represent a useful and appropriate educational tool. However, the quality of videos varied, and the level of information incompleteness was fairly high due to insufficient reviews. The role of private uploaders and academic institutions in surgical education cannot be overestimated. It is necessary that surgeon trainers and surgical educators critically analyze the quality of video content and exercise responsibility in directing trainee surgeons. In the current era, it is best for trainees to search for peer-reviewed content.

Keywords: Gastric cancer; laparoscopic gastrectomy; surgical training; YouTube; educational video

Submitted Oct 09, 2019. Accepted for publication Dec 24, 2019.

doi: 10.21037/atm.2020.01.78

View this article at: http://dx.doi.org/10.21037/atm.2020.01.78 


\section{Introduction}

Gastric cancer is a relatively prevalent malignancy and ranks fifth in diagnosed cancers and third in cancer-related deaths in the world (1). In 1994, Kitano first reported the technique for laparoscopic distal gastrectomy (LDG) (2). Since then, the use of laparoscopic gastrectomy (LG) for gastric cancer has increased due to its multiple advantages of improving patients' quality of life over open surgery. The rapid expansion of LG has generated interest among surgeons. Performing advanced laparoscopic surgery such as LG normally requires higher technique skills and a lengthy period of learning. Based on recent concerns, new learning tools are now required to overcome the constraints related to laparoscopic education. In contrast to open surgery, laparoscopy videos can provide surgical trainees with operating procedures and essential information on anatomy and operation technique. Operative videos are undoubtedly a useful and appropriate training tool for laparoscopic surgery.

Since 2005, YouTube has become the most visited video broadcast site on the internet. Over 1.9 billion logged-in users visit YouTube each month, and people watch over a billion hours of video and generate billions of views every day (3). Given that the online videos can be accessible anytime and anywhere on laptops, tablets, and smartphones, YouTube was considered a platform to provide medical information and education $(4,5)$. YouTube was the most commonly used video platform by trainees, with $95 \%$ of surgical residents using YouTube to prepare for surgery (5). However, videos can be uploaded from different sources and may be of varying quality. The videos posted on YouTube are not peer-reviewed and are normally ranked according to popularity, view counts, comments and user history, which are not valid criteria for videos with educational purposes.

Some studies have evaluated YouTube as a source of medical information. However, until now, no information is known about the characteristics of existing YouTube videos focusing on LG. The purpose of the present study was to evaluate the completeness and quality of LG videos for gastric cancer on the public video platform YouTube and to share our thoughts on important future directions for managing surgical videos about LG for gastric cancer.

\section{Methods}

We searched YouTube (www.youtube.com) on August 16, 2019 to locate video clips that included relevant information about LG for gastric cancer. The terms "laparoscopic gastrectomy" and "gastric cancer" were used to identify related video clips.

We performed our search and sorted the results according to three setting categories. The results were sorted by the website's default setting and view count separately. We also used length of duration to filter videos longer than 20 minutes, assuming that longer videos may have better educational value. The first 100 videos of each set of sorted results were gathered and analyzed.

Each video was viewed and analyzed for content. Any disagreements were resolved with consensus. Two experienced surgeons in the field of laparoscopic surgery reviewed videos together. Videos without demonstrations of laparoscopic technique (i.e., animations, lectures, patient experiences, news) were excluded.

We analyzed each video according to the following characteristics: length of duration, video provider, year of upload, number of views, video resolution, voice commentary, likes, and dislikes. Video provider was classified as academic, private, and other.

\section{Video Power Index (VPI)}

To evaluate the popularity of the videos, we use the "Video Power Index" (VPI) to assess both the view and the like ratio of the videos. The VPI was calculated as follows: first, calculate the like ratio (like*100/[like+dislike]) and the view ratio (number of views/days); then, the VPI is equal to the like ratio*view ratio/100 $(6,7)$.

\section{Fournal of American Medical Association benchmark criteria}

The Fournal of American Medical Association ( $7 A M A$ ) benchmark criteria was used to evaluate the basic quality and reliability of the videos $(6,7)$. The criteria consists of a 4-item (authorship, attribution, disclosure and currency) rating scale (Table 1). By assigning 1 point for the presence of each criterion, the total $7 A M A$ benchmark score was calculated (8).

\section{Completeness scores for $L G$}

At present, no validated tool for this purpose exists in the literature to provide a specific assessment of analyzed LG for gastric cancer-related videos. For a more detailed evaluation of the quality of videos, we used a completeness 
Table 1 The Fournal of American Medical Association ( $7 A M A)$ benchmark criteria

\begin{tabular}{ll}
\hline Criteria & Description \\
\hline Authorship & $\begin{array}{l}\text { Authors and contributors, their affiliations, and } \\
\text { relevant credentials should be provided }\end{array}$ \\
Attribution & $\begin{array}{l}\text { References and sources for all content should be } \\
\text { listed clearly, and all relevant copyright information } \\
\text { noted }\end{array}$ \\
Disclosure & $\begin{array}{l}\text { Web site "ownership" should be prominently } \\
\text { and fully disclosed, as should any sponsorship, } \\
\text { advertising, underwriting, commercial funding }\end{array}$ \\
Currency & $\begin{array}{l}\text { Dates that content was posted and updated } \\
\text { should be indicated }\end{array}$ \\
\hline
\end{tabular}

Table 2 Completeness checklist

\begin{tabular}{|c|c|}
\hline Contents & Score \\
\hline \multicolumn{2}{|l|}{ Videos resolution } \\
\hline High definition & 1 \\
\hline \multicolumn{2}{|l|}{ Preoperative evaluation } \\
\hline Age, gender, BMI & 1 \\
\hline Extent of gastrectomy & 1 \\
\hline Extent of LNs dissection & 1 \\
\hline Gl reconstruction method & 1 \\
\hline \multicolumn{2}{|l|}{ Procedure description } \\
\hline Words or voice comments & 1 \\
\hline \multicolumn{2}{|l|}{ During surgery } \\
\hline Port location & 1 \\
\hline \multicolumn{2}{|l|}{ LNs dissection } \\
\hline Infrapyloric LNs & 1 \\
\hline Suprapyloric LNs & 1 \\
\hline Great curvature LNs & 1 \\
\hline Suprapancreatic LNs & 1 \\
\hline Less curvature LNs & 1 \\
\hline \multicolumn{2}{|l|}{ Gl reconstruction } \\
\hline Stomach resection & 1 \\
\hline Anastomosis & 1 \\
\hline \multicolumn{2}{|l|}{ After surgery } \\
\hline Pathology stage & 1 \\
\hline Surgical outcomes & 1 \\
\hline
\end{tabular}

BMI, body mass index; LNs, lymph nodes; Gl, gastrointestinal. score that we developed (Table 2). The lymph node (LN) dissection was defined as the area of LNs that needed to be harvested when LG, including but was not limited to laparoscopic part gastrectomy (LPG), LDG, laparoscopic total gastrectomy (LTG), or laparoscopic proximal gastrectomy (LPG), was performed. The anatomical definitions of LN stations were as follows: infrapyloric LNs (No. 6), suprapyloric LNs (No. 5), along greater curvature LNs (No. 4, or plus 12a for LDG; No. 4, 2 or plus 12a for LTG), suprapancreatic LNs (No. 8a, 7, 9, or plus $11 \mathrm{p} / \mathrm{d}$ ), and along lesser curvature LNs (No. 1, 3).

\section{Statistical analysis}

Statistical analysis was performed with SPSS Version 23 software. Data were summarized as frequencies (n) and percentages (\%) for categorical variables and means or medians (standard deviations or ranges) for continuous and ordinal variables, respectively. Internal consistency between reviewers was evaluated with a kappa coefficient. The one-way ANOVA was used to compare the differences between the groups. A P value less than 0.05 was considered significant.

\section{Results}

\section{Video selection process}

In three search categories, we retrieved the first 100 videos. Some videos were duplicated in three different search categories. After duplicate videos were removed, 202 videos were selected for evaluation. Of the 202 videos screened, 102 videos met the inclusion criteria. The video selection process is shown in Figure 1.

\section{Video characteristics}

The characteristics of the analyzed videos are shown in Table 3. The mean length of duration for the videos was 5.1 minutes (range, 1.1-306.5), and the mean view count was 1,071 (range, 22-30,230). The mean video age was 2.9 years (range, 6 days to 10.2 years). Audio commentary was present in $33(32.4 \%)$ videos, among which English accounted for $72.7 \%$, followed by Bulgarian, accounting for $18.2 \%$. High definition (HD) resolution was provided in $53(52 \%)$ videos, most of which were created within the past 4 years. According to the video source, $71.6 \%$ of the videos were posted on YouTube by private users. Academic institutions 


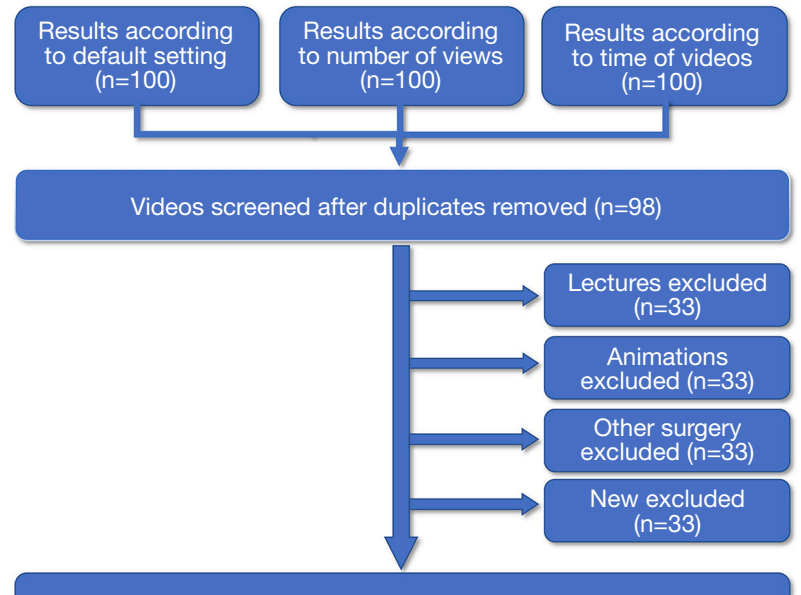

Full videos included and assessed in this study $(n=102)$

Figure 1 Flow diagram of video selection.

were responsible for uploading $19.6 \%$ of the total videos. The videos contributed by other sources, such as commercial companies, comprised only $8.8 \%$ of all videos.

\section{VPI}

The mean VPI score of all videos were 2.63 (range, 0 to 30.67). There were no differences based on whether the videos were sorted by source or content.

\section{$\mathcal{F A M A}$ benchmark scores}

The videos had a mean $7 A M A$ benchmark score of 1.94 (range, 1 to 4). There were 17 videos with 1 point, 75 videos with 2 points, 9 videos with 3 points and only 1 video with the full 4 points.

\section{Laparoscopic technique content}

In the evaluation based on surgical procedures, 49 (48\%) surgical procedures were LDG, 49 (48\%) were LTG, two were LPG and the remaining procedures could not be identified. Table 4 shows the exact contents of LDG and LTG. The extent of LN dissection was identified in 64 of the $102(62.7 \%)$ videos. Among the 64 videos, there were 59 for D2 gastrectomy. Among the LG procedures, LN dissection was the most frequently covered topic $(89.2 \%)$, followed in descending order by GI reconstruction (87.3\%). Twenty-four full-time videos without editing contain all technique details of LN dissection and GI reconstruction.
Table 3 Included videos characters

\begin{tabular}{|c|c|}
\hline Category & Number/median \\
\hline \multicolumn{2}{|l|}{ Video source } \\
\hline Academic institutions & $20(19.6 \%)$ \\
\hline Private users & $73(71.6 \%)$ \\
\hline Others & $8(8.8 \%)$ \\
\hline \multicolumn{2}{|l|}{ Cancer stage } \\
\hline Early stage & $23(22.5 \%)$ \\
\hline Advanced stage & $36(35.3 \%)$ \\
\hline Unclassified & $43(42.2 \%)$ \\
\hline \multicolumn{2}{|l|}{ Gastrectomy } \\
\hline Laparoscopic distal gastrectomy & $49(48.0 \%)$ \\
\hline Laparoscopic total gastrectomy & $49(48.0 \%)$ \\
\hline Laparoscopic proximal gastrectomy & $2(2.0 \%)$ \\
\hline Unknown & $2(2.0 \%)$ \\
\hline \multicolumn{2}{|l|}{ Lymphadenectomy } \\
\hline D1 & $3(2.8 \%)$ \\
\hline $\mathrm{D} 1+$ & $2(2.0 \%)$ \\
\hline D2 & $59(57.1 \%)$ \\
\hline $\mathrm{D} 2+$ & $1(0.9 \%)$ \\
\hline Unclassified & $38(37.2 \%)$ \\
\hline \multicolumn{2}{|l|}{ Origin } \\
\hline North America & $16(15.7 \%)$ \\
\hline Central and South America & $2(2.0 \%)$ \\
\hline Europe & $54(53.0 \%)$ \\
\hline South East & $8(7.8 \%)$ \\
\hline East Asia and Pacific & $14(13.7 \%)$ \\
\hline Middle East and Africa & $3(2.8 \%)$ \\
\hline Unknown & $5(5.0 \%)$ \\
\hline \multicolumn{2}{|l|}{ Videos resolution } \\
\hline Low definition & $48(47.1 \%)$ \\
\hline High definition & 54 (52.9\%) \\
\hline \multicolumn{2}{|l|}{ Video editing } \\
\hline Edited & $70(68.6 \%)$ \\
\hline Non-edited & $32(31.4 \%)$ \\
\hline \multicolumn{2}{|l|}{ Video characteristics } \\
\hline Number of days online & $1,089[6-3,710]$ \\
\hline Number of views & $1,071[22-30,230]$ \\
\hline Number of likes & 6 [0-99] \\
\hline Number of dislikes & $0[0-12]$ \\
\hline Video duration & $15.1[1.1-306.5]$ \\
\hline
\end{tabular}

Data are expressed as the number of cases (percentage) or median [range]. 
Table 4 Descriptive data of the videos

\begin{tabular}{|c|c|c|c|}
\hline Domain & LDG $(n=49)$ & LTG $(n=49)$ & $P$ value \\
\hline Number of views & $\begin{array}{c}4,972 \\
{[77-30,230]}\end{array}$ & $\begin{array}{c}2,907 \\
{[22-16,281]}\end{array}$ & 0.400 \\
\hline Technique description & & & 0.049 \\
\hline Yes & 15 (30.6\%) & $24(49.0 \%)$ & \\
\hline No & $34(69.4 \%)$ & $25(51.0 \%)$ & \\
\hline Lymphadenectomy & & & 0.460 \\
\hline D1 & 2 & 1 & \\
\hline D1+ & 1 & 0 & \\
\hline D2 & 27 & 27 & \\
\hline D2+ & 0 & 1 & \\
\hline Unclassified & 13 & 17 & \\
\hline No contents & 6 & 2 & \\
\hline \multicolumn{4}{|l|}{ Anastomosis } \\
\hline Billroth-I & 5 & - & - \\
\hline Billroth-II & 17 & - & \\
\hline Roux-en-Y & 13 & 45 & \\
\hline Overlap & - & 19 & \\
\hline Orvil $^{\mathrm{TM}}$ & - & 19 & \\
\hline Hand sewn & - & 3 & \\
\hline Reverse puncture & - & 2 & \\
\hline No contents & 13 & 6 & \\
\hline \multicolumn{4}{|l|}{ Information on scores } \\
\hline VPI & $3.23 \pm 6.56$ & $2.03 \pm 2.71$ & 0.241 \\
\hline JAMAS & $1.92 \pm 0.57$ & $1.96 \pm 0.54$ & 0.717 \\
\hline Completeness & $8.84 \pm 3.29$ & $9.73 \pm 2.32$ & 0.123 \\
\hline
\end{tabular}

LDG, laparoscopic distal gastrectomy; LTG, laparoscopic total gastrectomy.

The integrity of the video content was analyzed based on the surgical information completeness scores. Table 5 shows the information completeness scores.

\section{Video sources}

A total of $71.6 \%$ of the videos were posted on the YouTube by private users. Academic institutions were responsible for uploading $19.6 \%$ of the total videos (Table 6). Other sources, such as commercial companies, accounted for $8.8 \%$ of the videos. A total of 97 (95.1\%) videos with an identifiable
Table 5 Completeness score

\begin{tabular}{lcc}
\hline Completeness score & Number & Mean \pm SD \\
\hline Videos resolution & 54 & $0.54 \pm 0.50$ \\
Surgical information & 94 & $2.00 \pm 0.99$ \\
Procedure description & 42 & $0.41 \pm 0.49$ \\
Laparoscopic techniques & 102 & $5.64 \pm 2.19$ \\
Port location & 30 & $0.29 \pm 0.46$ \\
LNs dissection & 91 & $3.72 \pm 1.82$ \\
Gl reconstruction & 89 & $1.63 \pm 0.67$ \\
Surgical results & 31 & $0.48 \pm 0.78$ \\
Total (max =16) & 102 & $8.53 \pm 2.85$ \\
\hline
\end{tabular}

LNs, lymph nodes; Gl, gastrointestinal.

primary surgeon originated from eighteen different countries, most often from Bulgaria and the United States. Academic institutions (rather than other sources) tended to add demonstrations for each surgical procedure. The videos from academic institutions and private users were significantly more complete than those posted by other sources.

\section{Discussion}

During the past decade, many randomized controlled trials have confirmed that LG is safe and feasible and has many benefits, especially in postoperative recovery, compared with open gastrectomy. The percentage of laparoscopic procedures for gastric cancer is increasing, especially in the Asian region (9). Increasing interest in the LG procedure has forced people to obtain information over the internet (Figure 2). LG for gastric cancer normally includes complicated procedures and demands delicate and precise techniques. During surgical education, obvious difficulties may be associated with learning and practicing the theoretical and applied aspects of such minimally invasive techniques compared with traditional open gastrectomy. Trainee surgeons interested in such techniques can learn from training boxes, courses, conferences and even from online websites.

The incidence of gastric cancer is variable by region and culture. The incidence rate is markedly elevated in East Asia (1). The distribution of countries producing the analyzed videos differed from the distribution of countries based on gastric cancer incidence. More than $70 \%$ of videos came from Europe and Americas. However, only 13.7\% of 
Table 6 Sources and classification of detected videos

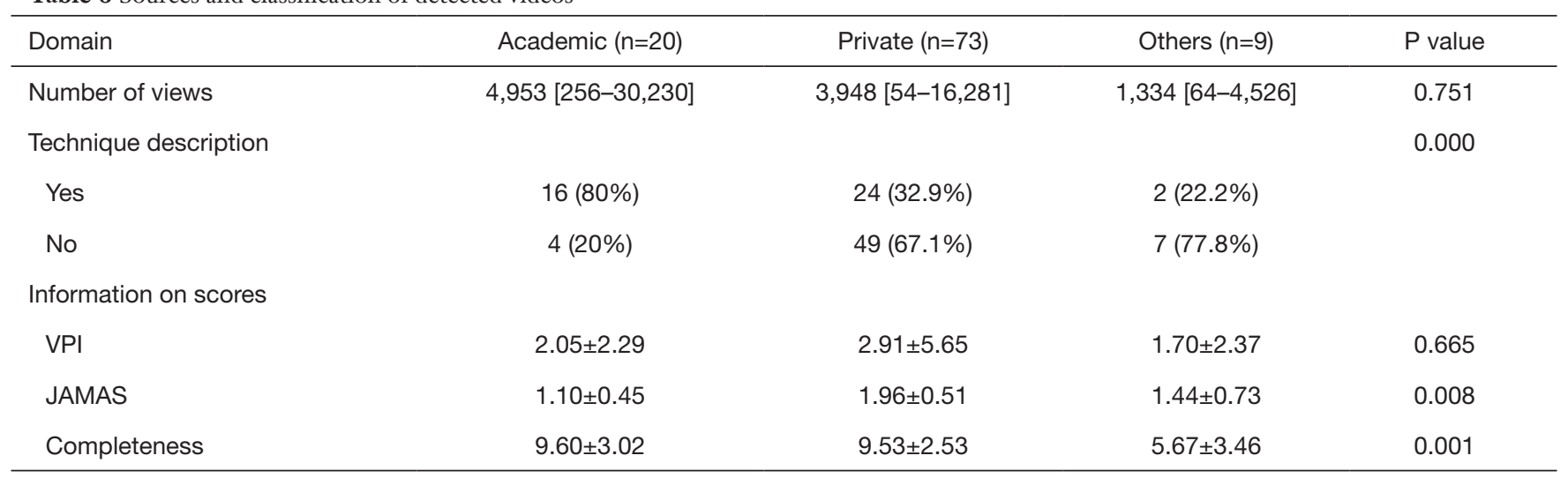

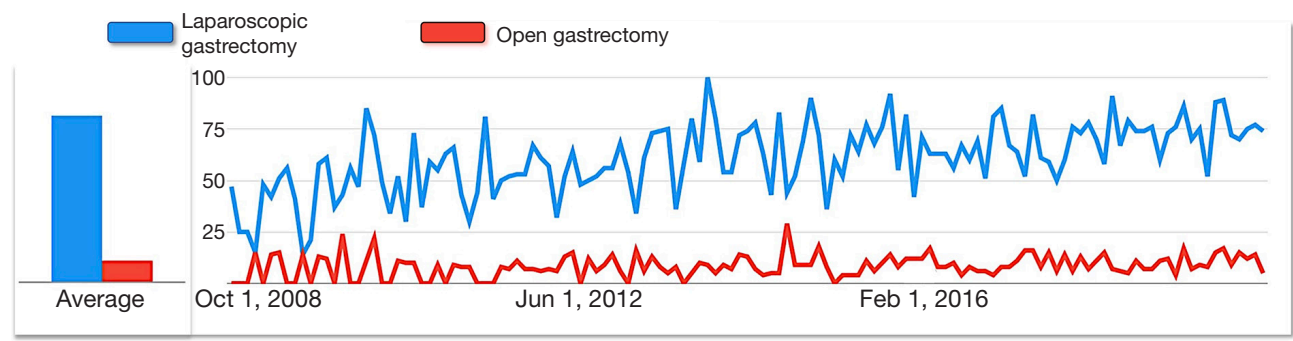

Figure 2 Increase in search keyword laparoscopic gastrectomy on Internet compared with open gastrectomy (https://trends.google.com).

videos came from East Asia. One reason for this may be that users in East Asia favored uploaded surgical videos using their native language (instead of English). Medical doctors in this region, who account for the majority of video sources, do not have time to pay sufficient attention to the platform because they frequently experience work overload and energy deficiencies (10-12), which seem to be another reason for this phenomenon.

The demonstration of each surgical procedure is critical because it is sometimes difficult for medical students or trainee surgeons to distinguish important anatomical structures and key steps of the surgical procedure from videos. Forty-one videos (40\%) included descriptions of surgical procedures, whether through the use of text, voice, or a combination with pictures. Academic institutions had the largest proportion (80\%) of technique descriptions compared with other sources. The results indicated that academic institutions may upload videos on YouTube primarily for educational purposes.

According to previous studies, the proportion of videos with HD resolution is usually less than $50 \%(13,14)$. Our results showed that approximately $52 \%$ of videos had HD resolution. However, we believe that the number of HD videos is still insufficient due to the complexity of the anatomical structures involved in LG and the dedicated skills required for appropriate technique. Excessive compression of image resolution can distort the view and reduce image quality to clinically unacceptable levels, which makes it difficult to distinguish important elements of the procedure.

When content was analyzed, videos on LDG and LTG each accounted for $48 \%$ of the total number of videos. LDG is the most frequently performed surgery among LG and is normally the first technique for trainee surgeons to learn. Three types of GI reconstruction are usually applied: gastroduodenostomy (Billroth I), loop gastrojejunostomy (Billroth II), and Roux-en-Y gastrojejunostomy. There is still no consensus regarding the best reconstruction method after distal gastrectomy $(15,16)$. The choice is usually dependent on tumor location and surgeons' preferences (17). Our results showed that Roux-en-Y was the most highly reconstructed method, followed by Billroth II. Roux-en-Y in LDG is a more complicated procedure than Billroth I or Billroth 
Table 7 Other English video-based education resources as an alternative to YouTube

\begin{tabular}{llll}
\hline Quality video-based education resource & Cost & Video platform & Website address \\
\hline WebSurg e-surgical reference & Free & Private platform & http://www.websurg.com \\
$\begin{array}{l}\text { American College of Surgeons (ACS) video library } \\
\text { Society of American Gastrointestinal and Endoscopic }\end{array}$ & Charge & Private platform & https://cine-med.com/acsonline \\
$\begin{array}{l}\text { Surgeons (SAGES) video library } \\
\text { Surgical Council on Resident Education (SCORE) }\end{array}$ & Based on YouTube & https://www.sages.org/video \\
MedTube & Charge & Private platform & https://portal.surgicalcore.org \\
\hline
\end{tabular}

II because it has two anastomoses: jejunojejunostomy and gastroduodenostomy. With the improvement of laparoscopic instruments and the continuous accumulation of surgical experience, the procedures can be performed for total laparoscopy. Our results showed that approximately $57.1 \%$ jejunojejunostomy was performed intracorporeally in videos with procedures of Roux-en-Y reconstruction. However, all gastroduodenostomy was performed by linear stapler intracorporeally. The proportion of LTG videos was $48 \%$ (the same as the proportion of LDG videos), which may indicate that most publishers thought that laparoscopy may be acceptable for total gastrectomy. The laparoscopic technique has been gradually adopted for total gastrectomy (18). However, LTG is still considered a very demanding procedure due to technical difficulties, especially esophago-jejunal reconstruction. The placement of anvil and intracorporeal purse-string sutures and anvil placement are technical challenges. The $\mathrm{OrVil}^{\mathrm{TM}}$ method by circular stapler and the overlap method by linear stapler are the most commonly used techniques for esophagojejunostomy. Our results showed that the OrVil ${ }^{\mathrm{TM}}$ method was applied in $42 \%$ of the videos, and the overlap method was also applied in $42 \%$ of the videos. There is still controversy about the reconstruction method (19). OrVil ${ }^{\mathrm{TM}}$ can reduce the difficulty of anvil placement but not tension through the use of a circular stapler. However, circular staplers are difficult to use, especially in obese patients with restricted space. The overlap method can overcome such an obstacle but requires a sophisticated suturing technique.

Our study demonstrated that 102 videos about LG were provided by different sources. Private users represent the greatest number of sources, followed by academic institutions. The video source may be related to the video quality, authenticity, and reliability. Academic institutions were considered to provide higher quality and valuable videos $(20,21)$. Most likely because of the professional field of LG, private uploaders in our study were usually surgeons who were proficient in the treatment of gastric cancer. The completeness scores did not differ between academic institutions and private users. To assess the popularity of videos about LG, we applied the VPI score. Our results show that private uploaders have a higher VPI. Watching operations performed by different surgeon pioneers around the world is an invaluable supplement to traditional education methods for laparoscopic surgery. However, we should note that surgeons may upload their laparoscopic videos on YouTube for reasons other than educational purposes. The total average VPI of the included videos was much lower than that of other kinds of surgical videos on YouTube $(7,22)$. One reason may be that people prefer to watch and learn simpler surgeries, such as laparoscopic cholecystectomy, on YouTube. The quality of laparoscopic videos varies and may limit the validity of the education provided. Because the validated scoring systems that are available do not reflect LG, we included a completeness score for LG. We found that the average completeness scores were only $54.1 \%$, with a combination of image quality, surgical information, procedure description, laparoscopic techniques and outcomes. Some videos only focused on part of the content of LG, such as the techniques of lymphadenectomy or reconstruction. It is unlikely to expect all videos to comprehensively cover all aspects of LG; it should be deemed that some videos, while incomplete, do contain precise and valuable content.

There is little doubt that YouTube is the largest video site in the world. However, there are now many other platforms that can provide educational surgical videos, both free-access and pay-per-view (Table 7). The videos are usually posted after academic review and quality control; a typical example is WebSurg. There were 29 videos about LG for gastric cancer by the end of September 2019. All the videos have voice commentaries and descriptions for each 
surgical procedure. The earliest videos of LG for gastric cancer were uploaded prior to those on YouTube (Table S1). These websites can be used as an alternative to YouTube.

The importance of online media in laparoscopic education cannot be overestimated. Surgeon trainers have been previously suggested that trainees should be taught "not what to know, but rather... how to acquire information, discriminate and make the right choice in the present moment" (23). This concept was expressed 15 years ago when the internet was not yet widely popular worldwide. However, it still seems to be representative today. It is necessary for trainer surgeons and surgical educators to exercise responsibility in directing trainee surgeons to use reliable resources.

There are limitations to our study. First, this study only comprises a snapshot of information when the study data were collected, and results may change due to new videos being added or removed with time. Second, we only selected YouTube to analyze laparoscopic videos, given that the website is reported as the largest and most popular video source. There may also be other surgical video websites organized by academic societies or commercial organizations.

\section{Conclusions}

Surgical videos about LG were largely provided by professionals, such as those in academic institutions and surgeons. The role of private uploaders and academic institutions in surgical education cannot be overestimated. The most covered techniques were LDG and LTG, which indicates that YouTube can serve as a useful and appropriate educational tool. However, the quality of videos varied, and the information incompleteness was fairly high due to insufficient reviews. It is necessary for trainer surgeons and surgical educators to critically analyze the quality of video content and to exercise responsibility in guiding trainee surgeons. In the current era, it is best for trainees to search for peer-reviewed content.

\section{Acknowledgments}

Funding: The study was supported by Japan China Sasakawa Medical Fellowship, and the China Scholarship Council (201908310012).

\section{Footnote}

Conflicts of Interest: The authors have no conflicts of interest to declare.

Ethical Statement: The authors are accountable for all aspects of the work in ensuring that questions related to the accuracy or integrity of any part of the work are appropriately investigated and resolved. This study did not require approval by the local Research Ethics Board as it involved publicly available data only.

Open Access Statement: This is an Open Access article distributed in accordance with the Creative Commons Attribution-NonCommercial-NoDerivs 4.0 International License (CC BY-NC-ND 4.0), which permits the noncommercial replication and distribution of the article with the strict proviso that no changes or edits are made and the original work is properly cited (including links to both the formal publication through the relevant DOI and the license). See: https://creativecommons.org/licenses/by-nc-nd/4.0/.

\section{References}

1. Bray F, Ferlay J, Soerjomataram I, et al. Global cancer statistics 2018: GLOBOCAN estimates of incidence and mortality worldwide for 36 cancers in 185 countries. CA Cancer J Clin 2018;68:394-424.

2. Kitano S, Iso Y, Moriyama M, et al. Laparoscopyassisted Billroth I gastrectomy. Surg Laparosc Endosc 1994;4:146-8.

3. Statistics. YouTube. 2019. Available online: http://www. youtube.com/yt/press/statistics.html. Accessed August 242019.

4. Petrucci AM, Chand M, Wexner SD. Social Media: Changing the Paradigm for Surgical Education. Clin Colon Rectal Surg 2017;30:244-51.

5. Rapp AK, Healy MG, Charlton ME, et al. YouTube is the Most Frequently Used Educational Video Source for Surgical Preparation. J Surg Educ 2016;73:1072-6.

6. Erdem MN, Karaca S. Evaluating the Accuracy and Quality of the Information in Kyphosis Videos Shared on YouTube. Spine (Phila Pa 1976) 2018;43:E1334-9.

7. Ferhatoglu MF, Kartal A, Ekici U, et al. Evaluation of the Reliability, Utility, and Quality of the Information in Sleeve Gastrectomy Videos Shared on Open Access Video Sharing Platform YouTube. Obes Surg 2019;29:1477-84.

8. Silberg WM, Lundberg GD, Musacchio RA. Assessing, controlling, and assuring the quality of medical information on the Internet: Caveant lector et viewor--Let the reader and viewer beware. JAMA 1997;277:1244-5. 
9. Shiroshita H, Inomata M, Bandoh T, et al. Endoscopic surgery in Japan: The 13th national survey (2014-2015) by the Japan Society for Endoscopic Surgery. Asian J Endosc Surg 2019;12:7-18.

10. Iwasaki K, Takahashi M, Nakata A. Health problems due to long working hours in Japan: working hours, workers' compensation (Karoshi), and preventive measures. Ind Health 2006;44:537-40.

11. Oh YI, Kim H, Kim K. Factors Affecting Korean Physician Job Satisfaction. Int J Environ Res Public Health 2019. doi: 10.3390/ijerph16152714.

12. Wu H, Liu L, Wang Y, et al. Factors associated with burnout among Chinese hospital doctors: a cross-sectional study. BMC Public Health 2013;13:786.

13. de'Angelis N, Gavriilidis P, Martinez-Perez A, et al. Educational value of surgical videos on YouTube: quality assessment of laparoscopic appendectomy videos by senior surgeons vs. novice trainees. World J Emerg Surg 2019; 14:22.

14. Toolabi K, Parsaei R, Elyasinia F, et al. Reliability and Educational Value of Laparoscopic Sleeve Gastrectomy Surgery Videos on YouTube. Obes Surg 2019;29:2806-13.

15. Lee MS, Ahn SH, Lee JH, et al. What is the best reconstruction method after distal gastrectomy for gastric cancer? Surg Endosc 2012;26:1539-47.

16. Zhang CD, Yamashita H, Seto Y. Gastric cancer surgery:

Cite this article as: Zhang S, Fukunaga T, Oka S, Orita H, Kaji S, Yube Y, Yamauchi S, Kohira Y, Egawa H. Concerns of quality, utility, and reliability of laparoscopic gastrectomy for gastric cancer in public video sharing platform. Ann Transl Med 2020;8(5):196. doi: 10.21037/atm.2020.01.78 historical background and perspective in Western countries versus Japan. Ann Transl Med 2019;7:493.

17. He Z, Zang L. Reconstruction after laparoscopic assisted distal gastrectomy: technical tips and pitfalls. Transl Gastroenterol Hepatol 2017;2:66.

18. Kodera Y, Yoshida K, Kumamaru H, et al. Introducing laparoscopic total gastrectomy for gastric cancer in general practice: a retrospective cohort study based on a nationwide registry database in Japan. Gastric Cancer 2019;22:202-13.

19. Kawamura H, Ohno Y, Ichikawa N, et al. Anastomotic complications after laparoscopic total gastrectomy with esophagojejunostomy constructed by circular stapler (OrVil()) versus linear stapler (overlap method). Surg Endosc 2017;31:5175-82.

20. Frongia G, Mehrabi A, Fonouni H, et al. You'Tube as a Potential Training Resource for Laparoscopic Fundoplication. J Surg Educ 2016;73:1066-71.

21. Lee JS, Seo HS, Hong TH. YouTube as a source of patient information on gallstone disease. World J Gastroenterol 2014;20:4066-70.

22. Aydin MA, Akyol H. Quality of Information Available on YouTube Videos Pertaining to Thyroid Cancer. J Cancer Educ 2019. [Epub ahead of print].

23. Satava RM. Disruptive visions: surgical education. Surg Endosc 2004;18:779-81. 
Supplementary

Table S1 LG videos on platform of WebSurg

\begin{tabular}{|c|c|}
\hline Category & Number/median \\
\hline \multicolumn{2}{|l|}{ Gastrectomy } \\
\hline LDG & $11(37.9 \%)$ \\
\hline LTG & $16(55.2 \%)$ \\
\hline LAPPG & $2(6.9 \%)$ \\
\hline \multicolumn{2}{|l|}{ Lymphadenectomy } \\
\hline D1 & $1(3.5 \%)$ \\
\hline $\mathrm{D} 1+$ & $4(13.8 \%)$ \\
\hline D2 & $11(37.9 \%)$ \\
\hline Unclassified & $13(44.8 \%)$ \\
\hline \multicolumn{2}{|l|}{ Country } \\
\hline Korea & $7(24.2 \%)$ \\
\hline Italy & $5(17.3 \%)$ \\
\hline France & $4(13.9 \%)$ \\
\hline Spain & $3(10.4 \%)$ \\
\hline Japan & 2 (6.9\%) \\
\hline Argentina & $2(6.9 \%)$ \\
\hline Belgium & $1(3.4 \%)$ \\
\hline Brazil & $1(3.4 \%)$ \\
\hline Portugal & $1(3.4 \%)$ \\
\hline United of Kingdom & $1(3.4 \%)$ \\
\hline Ireland & $1(3.4 \%)$ \\
\hline Switzerland & $1(3.4 \%)$ \\
\hline \multicolumn{2}{|l|}{ Videos resolution } \\
\hline Low definition & $3(10.3 \%)$ \\
\hline High definition & $26(89.7 \%)$ \\
\hline \multicolumn{2}{|l|}{ Video editing } \\
\hline Edited & $29(100 \%)$ \\
\hline \multicolumn{2}{|l|}{ Technique description } \\
\hline Voice commentary & $29(100 \%)$ \\
\hline \multicolumn{2}{|l|}{ Video characteristics } \\
\hline Number of days online & $2,293[70-4,900]$ \\
\hline Number of views & $3,953[1,202-10,745]$ \\
\hline Number of likes & $119[5-427]$ \\
\hline Number of dislikes & - \\
\hline Video duration & $17.3(6.7-306.5)$ \\
\hline
\end{tabular}

Data are expressed as the number of cases (percentage) or median (range). LG, laparoscopic gastrectomy; LDG, laparoscopic distal gastrectomy; LTG, laparoscopic total gastrectomy; LAPPG, laparoscopy-assisted pylorus-preserving gastrectomy. 